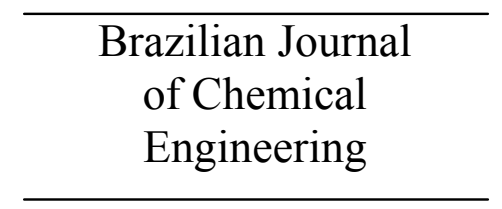

ISSN 0104-6632

Printed in Brazil

www.abeq.org.br/bjche

Vol. 29, No. 02, pp. 265 - 274, April - June, 2012

\title{
THE VISCOSITY DISTRIBUTION AROUND A RISING BUBBLE IN SHEAR-THINNING NON-NEWTONIAN FLUIDS
}

\author{
Shaobai $\mathrm{Li}^{1}$, Youguang $\mathrm{Ma}^{1 *}$, Taotao $\mathrm{Fu}^{1}$, Chunying $\mathrm{Zhu}^{1}$ and Huaizhi $\mathrm{Li}^{2}$ \\ ${ }^{1}$ State Key Laboratory of Chemical Engineering, School of Chemical Engineering and Technology, \\ Tianjin University, Tianjin 300072, China. \\ E-mail: ygma@tju.edu.cn \\ ${ }^{2}$ Laboratory of Reactions and Process Engineering, Nancy-Université, \\ CNRS, 1, rue Grandville, BP 20451, 54001 Nancy Cedex, France.
}

(Submitted: February 17, 2011 ; Revised: December 10, 2011 ; Accepted: December 29, 2011)

\begin{abstract}
The viscosity distribution of the liquid around a rising bubble in carboxymethyl cellulose (CMC) aqueous solutions was measured experimentally by particle image velocimetry (PIV). The effect of the concentration of CMC solutions on the viscosity distribution around a bubble and the coupling relations between the viscosity field, flow field and shear stress field were also studied. Results indicated that the specific viscosity (non-dimensionalized by $\eta_{0}$ ) decreases with the increase in CMC solution concentration due to a shear thinning effect. Within the experimental range, similar viscosity distributions of liquids around a rising bubble were found: a hollow cylindrical low viscosity region around the bubble wake and a high viscosity region in the central bubble wake.

Keywords: Non-Newtonian fluid; Bubble; Viscosity distribution; Shear-thinning effect.
\end{abstract}

\section{INTRODUCTION}

Gas-liquid two phase flow is frequently encountered in many industrial fields such as mineral, chemical, food, biochemical and waste water treatment. The underlying knowledge of bubbles moving in fluids is an essential foundation for the design and optimization of process units (Clift et al., 1978). In recent decades, studies on bubble behavior in gas-liquid two-phase flow have attained remarkable achievements both experimentally and theoretically. Davies et al. (1950) observed the shape and velocity of air bubbles varying with volume in water. Lance $e t$ al. (1991) studied the turbulence induced by bubbles in an air-water system. Chen et al. (1998) observed the wake of bubbles in water. Hassan et al. (2001) studied the velocity field of the bubble and Rodrigue
(2004) proposed a general correlation for the drag coefficient of bubbles. Ribeiro Jr. et al. (2004) studied bubble size distributions and bubble mean diameters by a photographic technique for a direct-contact evaporator operating. Kulkarni et al. (2005) presented a comprehensive review of bubble behavior in gasliquid systems and illustrated the present status of the subject thoroughly. Mandal (2010) studied the gas holdup, bubble sizes and their distribution for waterair system in a down-flow jet loop reactor. However, in comparison with Newtonian fluids, the understanding of the moving behavior and mass transfer mechanism of bubbles in non-Newtonian fluids remains still insufficient due to the inherent complexities.

It has been found that the bubble shape and rising velocity (Moore, 1958), the interaction between

*To whom correspondence should be addressed 
bubbles (Toshiyuki et al., 2005), and the interphase mass and heat transfer (Wang et al., 2010) depend dramatically on the viscosity of the liquid. In addition, for non-Newtonian fluids, the viscosity is also susceptible to the shear stress due to the complex rheological behavior. Shear thinning nonNewtonian fluids are the most common kind of nonNewtonian fluids and are characterized by a decreasing apparent viscosity with increasing shear rate. This kind of non-Newtonian fluid is widely used in industry. For instance, the fermentation liquor in many biochemical processes is a typical shear thinning non-Newtonian fluid. Because high shear stress can demolish the fragile microorganisms, the study of the relationship between the viscosity and the shear stress is an essential prerequisite and could provide useful information for biochemical process design and optimization (Zhao et al., 1998; Al-Masry, 1999). Allen et al. (1991) studied the mean apparent viscosity of fermentation liquor in a bubble column bioreactor and found that the viscosity of the fermentation liquor was proportional to the superficial gas velocity. Kawase et al. (1991) introduced the concept of energy dissipating rate and concluded that the mean apparent viscosity of fermentation liquor relied strongly on the energy dissipating rate, superficial gas velocity and rheological properties of the liquid. Due to the limitation of measurement techniques, the above mentioned studies mostly considered the mean apparent viscosity of non-Newtonian fluids. However, when a bubble rises in a non-Newtonian fluid, the local viscosity of the liquid near a rising bubble is quite different and depends on the shear stress distribution caused by the relative motion of fluids. Therefore, information on the local viscosity distribution around rising bubbles is necessary for the better understanding of the bubble moving behavior in non-Newtonian fluids. Unfortunately, although Zhang et al. (2010) numerically explored the local viscosity distribution around bubbles in shear-thinning non-Newtonian fluids using the level set approach, so far there is little experimental information about the local viscosity distribution around bubbles rising in non-Newtonian fluids.

As a typical non-Newtonian fluid, carboxymethyl cellulose (CMC) solutions can model various types of non-Newtonian fluids used in many industrial processes due to their shear-thinning property. In this work, the local viscosity distribution of the liquid around a rising bubble in an aqueous $\mathrm{CMC}$ solution was studied using particle image velocimetry (PIV).

\section{PROCEDURE}

\section{Experimental Apparatus}

The schematic diagram of the experimental apparatus is shown in Figure 1. Gas was injected via a nozzle (inner diameter: $1.0 \mathrm{~mm}$ ) into the bottom of a bubble column $(150 \mathrm{~mm} \times 150 \mathrm{~mm} \times 1700 \mathrm{~mm})$ from a gas cylinder by a valve and rotameter (within \pm 0.01 $\mathrm{cm}^{3} / \mathrm{s}$ ). The time interval between successive bubbles was long enough $(120 \mathrm{~s})$ to minimize the mutual influence between them. The flow field was measured by particle image velocimetry (PIV) (3D FlowMaster, LaVision GmbH, Germany) which consisted of a double pulsed Nd:YAG laser (532 nm wavelength), a high-resolution CCD camera (LaVision FlowMaster 3 system, image size $1376 \times 1040$ ) with a Nikon lens. The appropriate amount of fluorescent tracer particles was seeded into the fluid homogeneously. These fluorescent tracer particles have a density of 1050 $\mathrm{kg} \cdot \mathrm{m}^{-3}$ and a mean diameter of $7 \mu \mathrm{m}$ that can follow the flow closely and have nearly no effect on the flow structure and fluid properties. The beam from the laser passed through the laser lens system, forming a sheet of light that illuminated the observing field ( 80 $\mathrm{mm} \times 60 \mathrm{~mm}) 700 \mathrm{~mm}$ above the nozzle at the double pulsed intervals of $100 \mu \mathrm{s}$. Two successive exposures of the flow to the pulsed laser light sheet were recorded in two separate frames. Steady motion of the bubbles is reached after moving a distance equivalent to a few bubble diameters, so the bubbles rise at their terminal velocity within the observing field. The camera recorded 5 pairs of frames per second. Typical images with tracer particles and the bubbles' real shapes are shown in Figure 2. These images were divided into small interrogation windows the size of $16 \times 16$ pixels with an interrogation area overlap of $50 \%$. The data processing was completed with LaVision's software (Flow Master 3) using the crosscorrelation algorithm. The main error source in the velocity measurements was the displacement of the particles in the image between two consecutive frames, which depended on the optical parameters of the camera, lenses and laser and the algorithm for finding the centroid of the particles in the image. All experiments were carried out at room temperature and atmospheric pressure. 


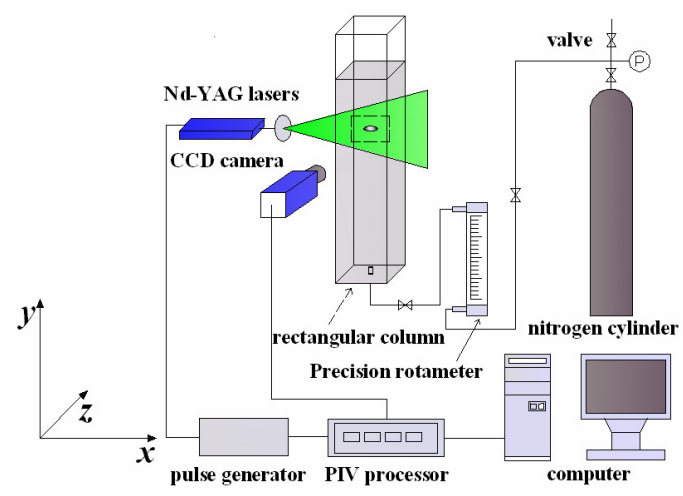

Figure 1: Schematic diagram of the experimental setup.

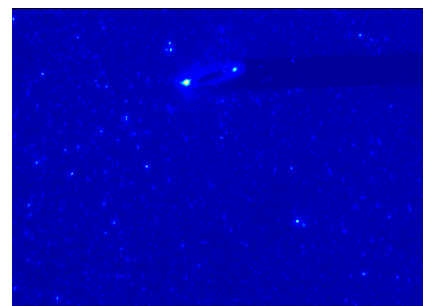

(a)

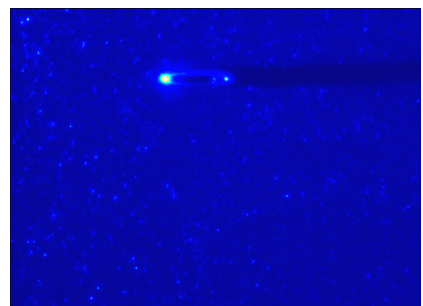

(b)

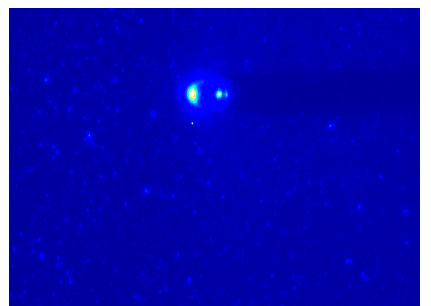

(c)

Figure 2: The images of bubbles captured by PIV. (a) $0.2 \% \mathrm{CMC}$; (b) $0.6 \% \mathrm{CMC}$; (c) $1.0 \% \mathrm{CMC}$.

Nitrogen was used as the gas phase and its density and viscosity are $1.25 \mathrm{~kg} \cdot \mathrm{m}^{-3}$ and $1.7 \times 10^{-5} \mathrm{~Pa} \cdot \mathrm{s}$, respectively. An aqueous solution of $\mathrm{CMC}$ (analytical grade; Mw: $5 \times 10^{6} \mathrm{D}$; Tianjin Kermel Chemical Reagent Co.,Tianjin, China) was used as the liquid phase. Mass concentrations of the aqueous CMC solutions were $0.2 \%, 0.6 \%$, and $1.0 \%$, respectively. The density of the liquid was measured using a densimeter (AntonPaar, DMA5000, Austria) with an accuracy of $\pm 1.0 \%$. The surface tension was measured with a dynamic surface tension apparatus (DCAT21, dataphysics, Germany) with an accuracy of $\pm 1.0 \%$. Liquid physical properties and bubble parameters are listed in Table 1.

Table 1: Liquid physical properties and parameters of bubbles in the experiments.

\begin{tabular}{|l|c|c|c|c|}
\hline Fluid & $\boldsymbol{\rho}\left(\mathbf{k g} \cdot \mathbf{m}^{-\mathbf{3}}\right)$ & $\boldsymbol{\sigma}\left(\mathbf{m} \mathbf{N} \cdot \mathbf{m}^{-\mathbf{1}}\right)$ & $\mathbf{d}_{\mathbf{e}}(\mathbf{m m})$ & $\mathbf{U}\left(\mathbf{m} \cdot \mathbf{s}^{-\mathbf{1}}\right)$ \\
\hline $0.2 \%$ CMC & 1001 & 63.47 & 8.0 & 0.21 \\
$0.6 \%$ CMC & 1007 & 55.73 & 8.2 & 0.18 \\
$1.0 \%$ CMC & 1015 & 52.55 & 8.1 & 0.16 \\
\hline
\end{tabular}

\section{Computational Method}

A commercial program, TECPLOT, was used for post-processing of the PIV data. In order to obtain the viscosity distribution around a rising bubble, the recorded instantaneous flow field around the bubble is divided into a series of grids to execute the interpolation, resulting in a grid size of $1.0 \mathrm{~mm} \times 1.0 \mathrm{~mm}$. The interpolation is a method of constructing new data points within the range of a discrete set of known data points. Here, in executing the interpolation process, the Kriging algorithm was performed for the instantaneous flow field. For aqueous CMC solutions, the viscosity $\eta(\dot{\gamma})$ depends on the shear rate $\dot{\gamma}$. The shear rate $\dot{\gamma}$ of each grid point is calculated based on a finitedifference approximation for the interpolated velocity field as follows:

$$
\begin{aligned}
\dot{\gamma}= & \frac{\Delta \mathrm{U}_{\mathrm{x}}}{\Delta \mathrm{y}}+\frac{\Delta \mathrm{U}_{\mathrm{y}}}{\Delta \mathrm{x}}=\frac{\mathrm{U}_{\mathrm{x}}(\mathrm{i}, \mathrm{j}+1)-\mathrm{U}_{\mathrm{x}}(\mathrm{i}, \mathrm{j}-1)}{2 \Delta \mathrm{y}}+ \\
& \frac{\mathrm{U}_{\mathrm{y}}(\mathrm{i}+1, \mathrm{j})-\mathrm{U}_{\mathrm{y}}(\mathrm{i}-1, \mathrm{j})}{2 \Delta \mathrm{x}}
\end{aligned}
$$

Here $U_{x}(i, j+1)$ and $U_{x}(i, j-1)$ are the velocity components in the $x$ direction of the calculated grid points $(i, j+1)$ and $(i, j-1)$, respectively. $U_{y}(i+1, j)$ and $\mathrm{U}_{\mathrm{y}}(\mathrm{i}-1, \mathrm{j})$ are the velocity components in the $\mathrm{y}$ direction of the grid points $(i+1, j)$ and $(i-1, j)$, respectively. $\Delta \mathrm{x}$ and $\Delta \mathrm{y}$ are the lengths in the $\mathrm{x}$ and $\mathrm{y}$ directions of the calculated grid point $(i, j)$, respectively. The viscosity of CMC solutions could 
be calculated by a suitable constitutive equation according to the shear rate $\dot{\gamma}$. In order to describe the shear-thinning degree of different concentrations of CMC solutions, the viscosity is represented by the specific viscosity (made dimensionless by dividing by $\eta_{0}$, where $\eta_{0}$ is the viscosity corresponding to zero shear rate, which expresses the viscosity of quiescent non-Newtonian fluids):

$$
\eta=\eta(\dot{\gamma}) / \eta_{0}
$$

The shear stress can be described by the product of viscosity $\eta(\dot{\gamma})$ and shear rate $\dot{\gamma}$.

$$
\tau=\eta(\dot{\gamma}) \cdot \dot{\gamma}
$$

\section{RESULTS AND DISCUSSION}

\section{Rheological Properties of the Liquid}

As is well known, it is impossible to establish a general constitutive equation for all kinds of nonNewtonian fluids. Therefore, selecting a proper constitutive equation for a specific non-Newtonian fluid is necessary. Among the existing constitutive equations, the simplest and most widely used one is the power-law model (Schowalter, 1960):

$$
\eta(\dot{\gamma})=K(\dot{\gamma})^{\mathrm{n}-1}
$$

$\mathrm{K}$ is the consistency index and $\mathrm{n}$ the flow index, which indicates the degree of deviation from a Newtonian fluid; the fluid is a Newtonian fluid when $n=1$.

Another extensively used constitutive equation is the Carreau model (Carreau, 1972):

$$
\eta(\dot{\gamma})=\eta_{0}\left[1+(\lambda \dot{\gamma})^{2}\right]^{(\mathrm{s}-1) / 2}
$$

where $\eta_{0}$ is the zero-shear viscosity, $\lambda$ the characteristic time of the liquid, and $s$ the power law index. The Carreau model is a three parameters model and can be used at low shear rate.

The rheological properties of CMC solutions were measured using a viscosimeter (Brookfield, DV-III, USA) at the experimental temperature. The measuring accuracy was $\pm 1.0 \%$ within the measuring range and the shear rate range was $0.1 \sim 150 \mathrm{~s}^{-1}$. The change of apparent viscosity along with shear rate for CMC solutions is shown in Figure 3. The predictions of the power-law model and Carreau model are plotted as dotted and solid lines, respectively, and the parameters of equations were determined by fitting the shear stress and shear rate data (Table 2). It can be clearly seen from Figure 2 that the results predicted by the Carreau model exhibit good agreement with the experimental data. Accordingly, the Carreau model was adopted to characterize the shear thinning behavior of $\mathrm{CMC}$ solutions in this work.

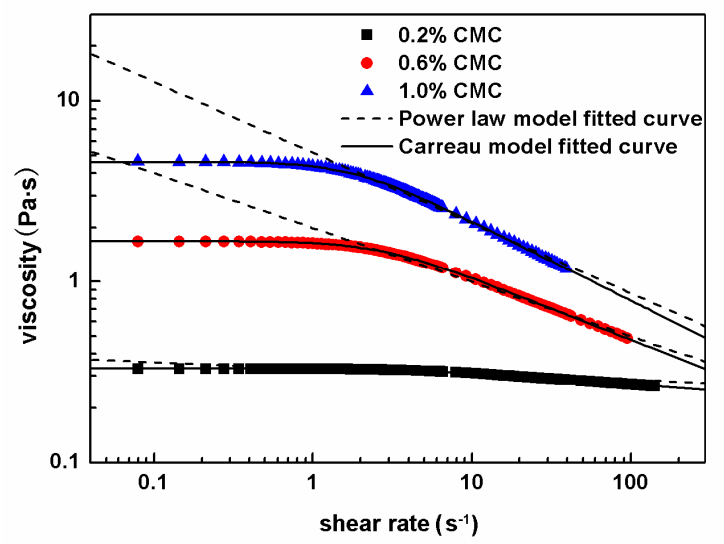

Figure 3: Shear viscosity as a function of the shear rate for different $\mathrm{CMC}$ solutions.

Table 2: Rheological parameters of different percentages of CMC solutions.

\begin{tabular}{|l|c|c|c|c|c|}
\hline Fluid & $\mathbf{K}\left(\mathbf{P a} \cdot \mathbf{s}^{\mathbf{n}}\right)$ & $\mathbf{n}$ & $\boldsymbol{\eta}_{\mathbf{0}}(\mathbf{P a} \cdot \mathbf{s})$ & $\boldsymbol{\lambda}(\mathbf{s})$ & $\mathbf{s}$ \\
\hline $0.2 \% \mathrm{CMC}$ & 0.3306 & 0.9675 & 0.3301 & 0.2508 & 0.9377 \\
$0.6 \% \mathrm{CMC}$ & 1.9241 & 0.6963 & 1.6831 & 0.3826 & 0.6544 \\
$1.0 \% \mathrm{CMC}$ & 5.1421 & 0.5845 & 4.6234 & 0.5568 & 0.5593 \\
\hline
\end{tabular}

\section{Flow Field Around a Bubble}

The non-uniformity of the liquid flow field near a rising bubble is responsible for the viscosity unevenness at each local point around the bubble in a non-Newtonian fluid due to the shear-thinning effect. Information on the flow field is accordingly an important basis for a the better understanding of the local viscosity distribution around a rising bubble. Figure 4 illustrates the measured flow field distributions around rising bubbles at different concentrations of $\mathrm{CMC}$, where white represents the shape of the bubble. Figure 4 shows that the flow field displays a typical structure: the rising bubble pushes the liquid ahead of it in up-flow and the liquid on both sides of the bubble flows back into the bubble wake, forming a pseudo-plug flow, as reported by Funfschilling et al. (2001). The bubble follows a spiral trajectory at low concentration of CMC solution, as shown in Figure 4(a). The reason for the spiral trajectory of the bubbles is the unstable wake of the bubble, as reported by Zenit et al. (2009). The unstable wake induces a horizontal 
force, causing the sideways motion, which leads to the path instability of the bubble. It also can be seen from Figure 4(a) that a spiral vortex rope appears around the wake of the bubble. Brücker (1999) pointed out that there exist two components of the lift force due to the specific pressure field induced by the oscillation of the bubble surface; one moves the bubble laterally and the other keeps the bubble on a circular path, leading to the occurrence of the spiral vortex rope. When the concentration of CMC solution increases, the velocity of the bubble decreases and the rising trajectory of the bubble varies from spiral to rectilinear. Consequently, the spiral vortex rope disappears and a vortex ring appears around the wake of the bubble. The vortex ring follows the bubble due to the upward pressure induced by the velocity gradient around it. At the same time, the bubble shape evolves from oblate to spherical, as shown in Figure 4(b) and 4(c). The instantaneous liquid velocity distributions $\left(y / d_{e}=0\right.$ and -2$)$ in the $\mathrm{x}$-direction and $\mathrm{y}$-direction are shown in Figure 5(a) and 5(b), respectively, adopting the center of the bubble bottom as the origin of the coordinates. The velocity components in the $\mathrm{x}$ direction and y-direction decrease gradually with the increase of the concentration of the CMC solution, but the velocity gradient in the $y$-direction increases gradually with the increase of the CMC solution concentration because high concentrations of CMC result in high viscosity, which leads to a large energy dissipation rate and prevents the momentum from transfering to far away regions.

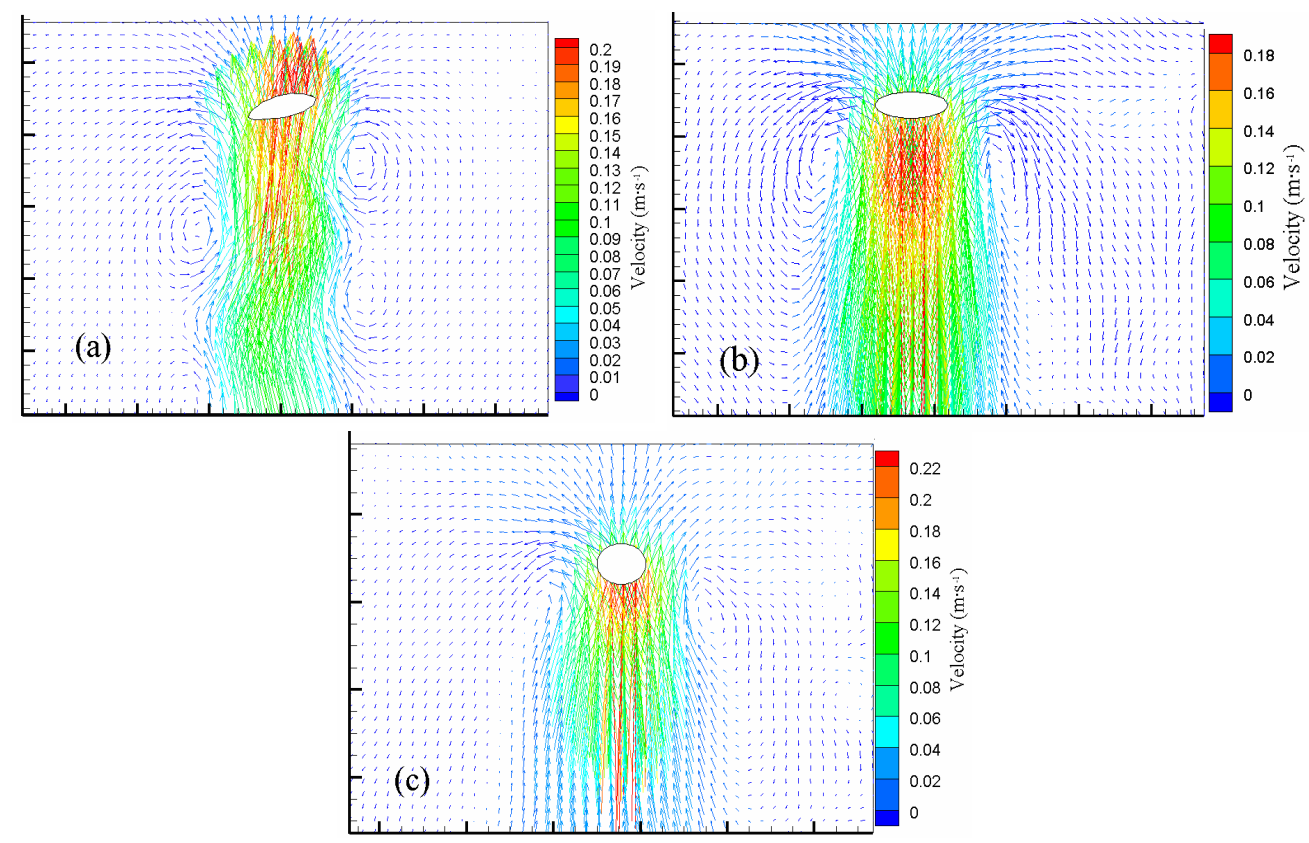

Figure 4: Flow field and shape of bubble rising in CMC solutions (m/s). (a) $0.2 \% \mathrm{CMC}, \mathrm{d}_{\mathrm{e}}=8.0 \mathrm{~mm}$; (b) $0.6 \%$ CMC, $\mathrm{d}_{\mathrm{e}}=8.2 \mathrm{~mm}$; (c) $1.0 \% \mathrm{CMC}, \mathrm{d}_{\mathrm{e}}=8.1 \mathrm{~mm}$.
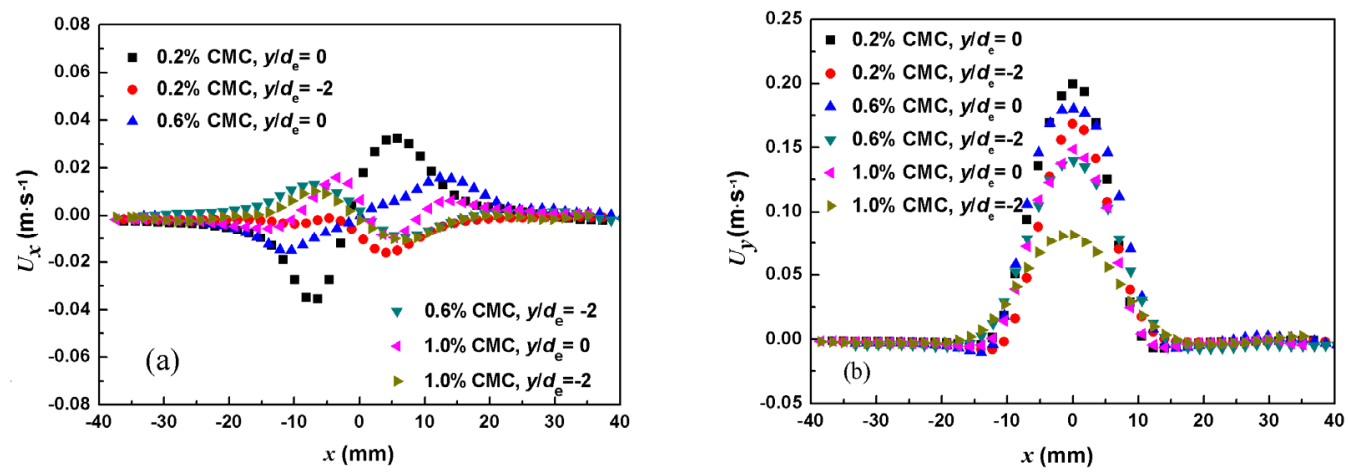

Figure 5: Instantaneous velocity components of the liquid at $y / d_{e}=0$ and $y / d_{e}=-2$. (a) $U_{x}$-component in the $\mathrm{x}$-direction; (b) $\mathrm{U}_{\mathrm{y}}$-component in the $\mathrm{y}$-direction. 


\section{Viscosity Field Around a Rising Bubble}

When a single bubble rises in a shear-thinning fluid, liquid motion results in the change of viscosity of the liquid around the bubble due to different shear stress effect. Otherwise, the variation of the viscosity would lead to a change in the velocity and shape of the bubbles. Figure 6 illustrates the experimental results for the viscosity field around a single bubble rising in solutions with different concentrations of CMC. Figure 6(b) and Figure 6(c) indicate that the viscosity distributions of the liquid near the bubble are similar: a hollow cylindrical low viscosity region around the wake of the bubble and a cylindrical high viscosity region in the central wake. This result also shows good conformity to the simulated results (Zhang et al., 2010). Moreover, because the viscosity distribution around a bubble is closely related to the flow field, as shown in Figure 4, the liquid behind the bubble has a high flow velocity and low viscosity, while the wake presents pseudo-plug flow. The high shear rate corresponds to the low viscosity region, while the low shear rate forms a high viscosity region. The minimum specific viscosity decreases with the increase of the concentration of $\mathrm{CMC}$ solution. For mass concentrations of $0.2 \%, 0.6 \%$ and $1.0 \%$, the minimum specific viscosities are $0.89,0.5$ and 0.4 , respectively. This can be attributed to the shear thinning effect of CMC solutions, because when the concentration of CMC solutions increases, both the flow index $\mathrm{n}$ and the minimum of specific viscosity decrease, as shown in Figure 6(a). As shown in Figure 6(a), the viscosity distribution in $0.2 \% \mathrm{CMC}$ solutions is very different from that at high concentration of CMC. The bubble rose with a spiral trajectory in $0.2 \% \mathrm{CMC}$ solution, leading to the appearance of a spiral vortex rope around the bubble wake. The large relative velocity between the liquid around the vortex rope and the bulk liquid led to the occurrence of a large shear stress, which resulted in a low viscosity region in a spiral rope form. Simultaneously, there existed a spiral high viscosity region in the center of the bubble wake. In Figure 7(a), the radial viscosity distribution decreased alternately due to the spiral rise of the bubble, but in Figure 7(b) and 7(c) presented a perfect symmetry with the trajectory of the bubble motion gradually tending to linear.

\section{Shear Behavior Around a Bubble}

The shear behavior is principally responsible for the viscosity change of a non-Newtonian fluid. Therefore, the shear behavior is directly reflected in the viscosity distribution. Figure 8 and Figure 9 show the shear stress fields and shear rate fields around a single bubble rising in solutions with different concentrations of CMC. Note that the shear stress fields are the same as the shear rate fields and all display a butterfly-like spatial structure similar to the viscosity field distribution shown in Figure 6. This structure is also consistent with the visualization by the birefringence method (Funfschilling et al., 2001). The maximal shear stress and the shear rate increase with increasing $\mathrm{CMC}$ concentration. The higher shear behavior region corresponds to the lower viscosity region, and vice versa. There exists a higher shear region around the wake due to the vortex ring structure of the flow field of the liquid, in this region, the effect of shear-thinning becoming very strong.

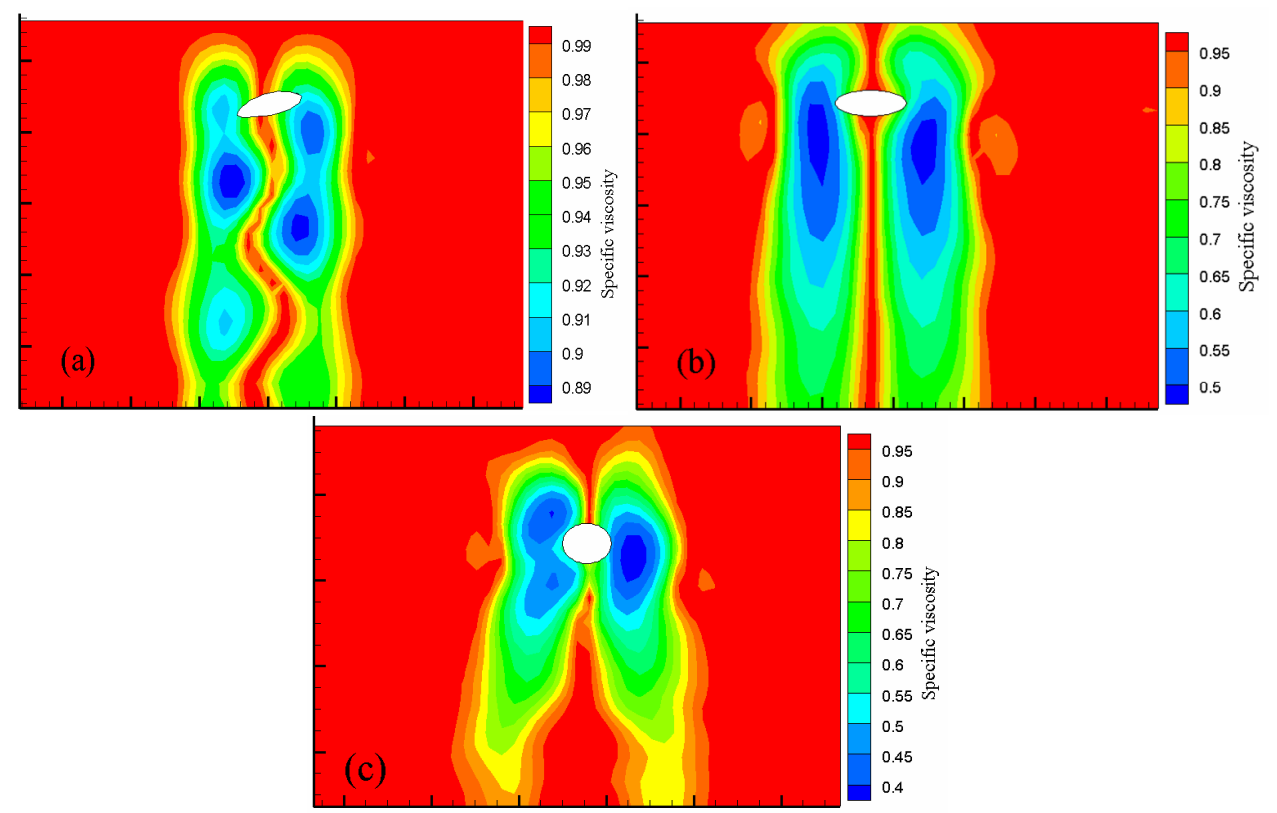

Figure 6: Viscosity field around a single bubble rising in CMC solutions. (a) $0.2 \% \mathrm{CMC}, \mathrm{d}_{\mathrm{e}}=8.0 \mathrm{~mm}$; (b) $0.6 \%$ CMC, $\mathrm{d}_{\mathrm{e}}=8.2 \mathrm{~mm}$; (c) $1.0 \mathrm{CMC}, \mathrm{d}_{\mathrm{e}}=8.1 \mathrm{~mm}$. 

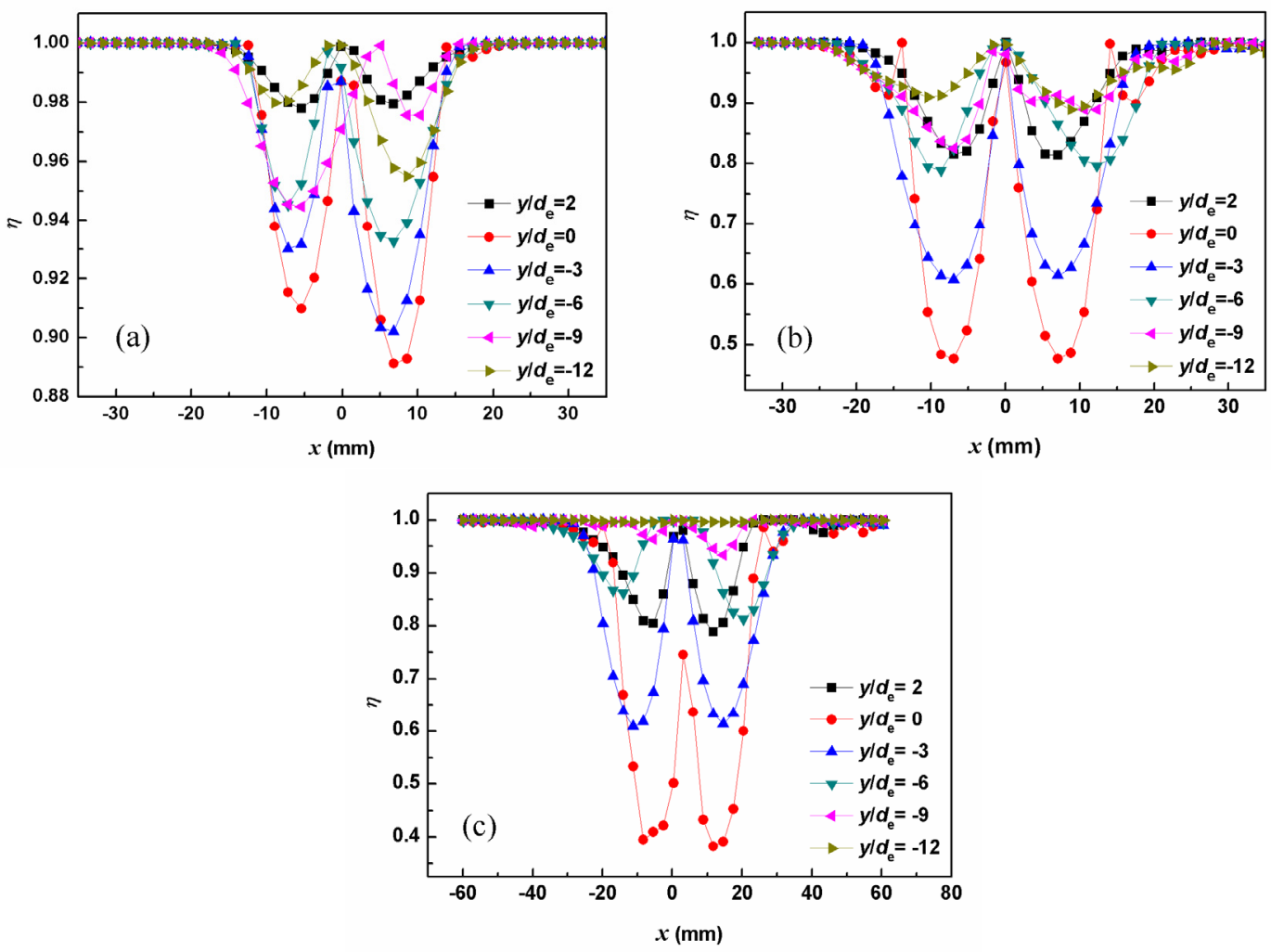

Figure 7: Effects of the concentration of CMC solutions on the viscosity distribution. (a) $0.2 \% \mathrm{CMC}$, $\mathrm{d}_{\mathrm{e}}=8.0 \mathrm{~mm}$; (b) $0.6 \% \mathrm{CMC}, \mathrm{d}_{\mathrm{e}}=8.2 \mathrm{~mm}$; (c) $1.0 \% \mathrm{CMC}, \mathrm{d}_{\mathrm{e}}=8.1 \mathrm{~mm}$.
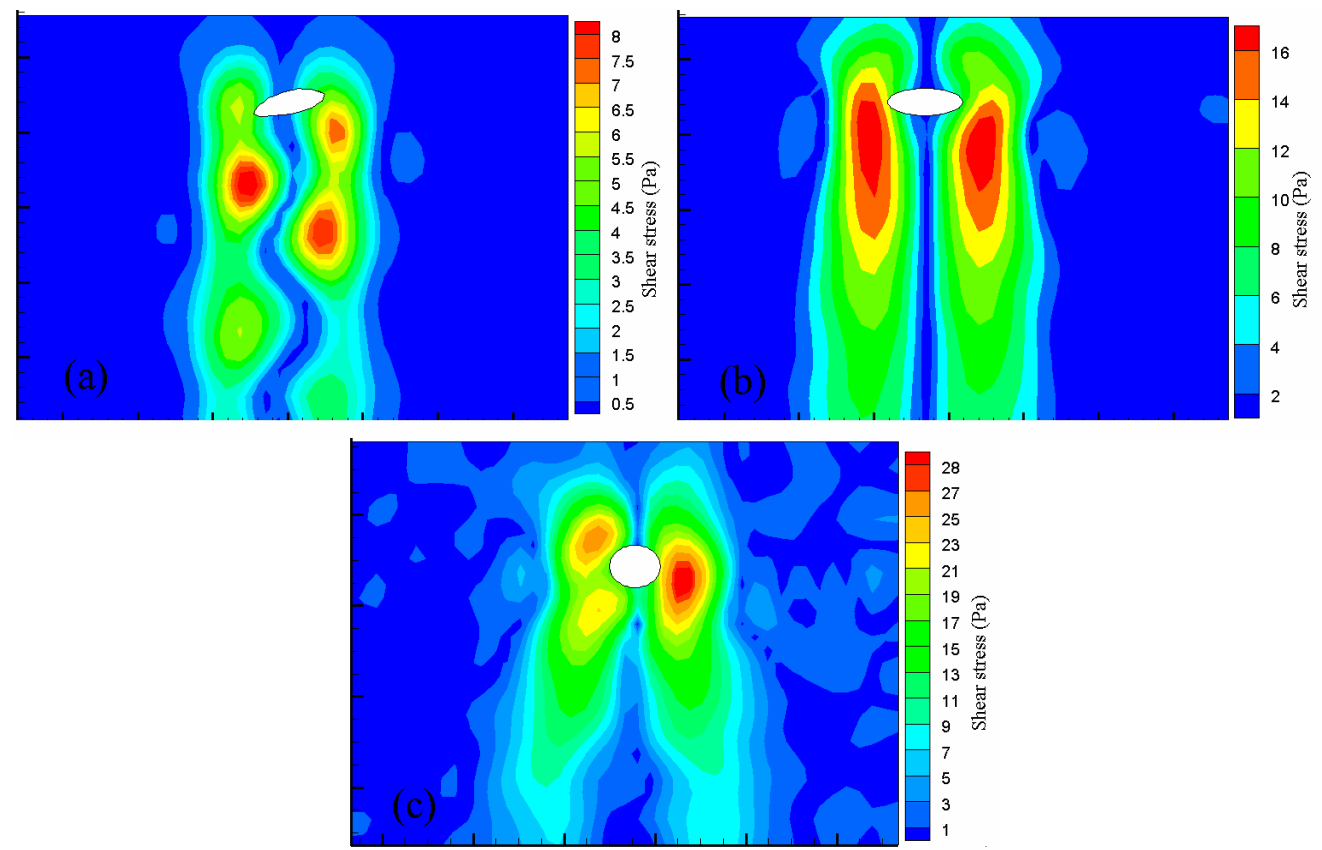

Figure 8: Shear stress distribution in CMC solutions. (a) $0.2 \% \mathrm{CMC}, \mathrm{d}_{\mathrm{e}}=8.0 \mathrm{~mm}$; (b) $0.6 \% \mathrm{CMC}, \mathrm{d}_{\mathrm{e}}=8.2 \mathrm{~mm}$; (c) $1.0 \% \mathrm{CMC}, \mathrm{d}_{\mathrm{e}}=8.1 \mathrm{~mm}$. 

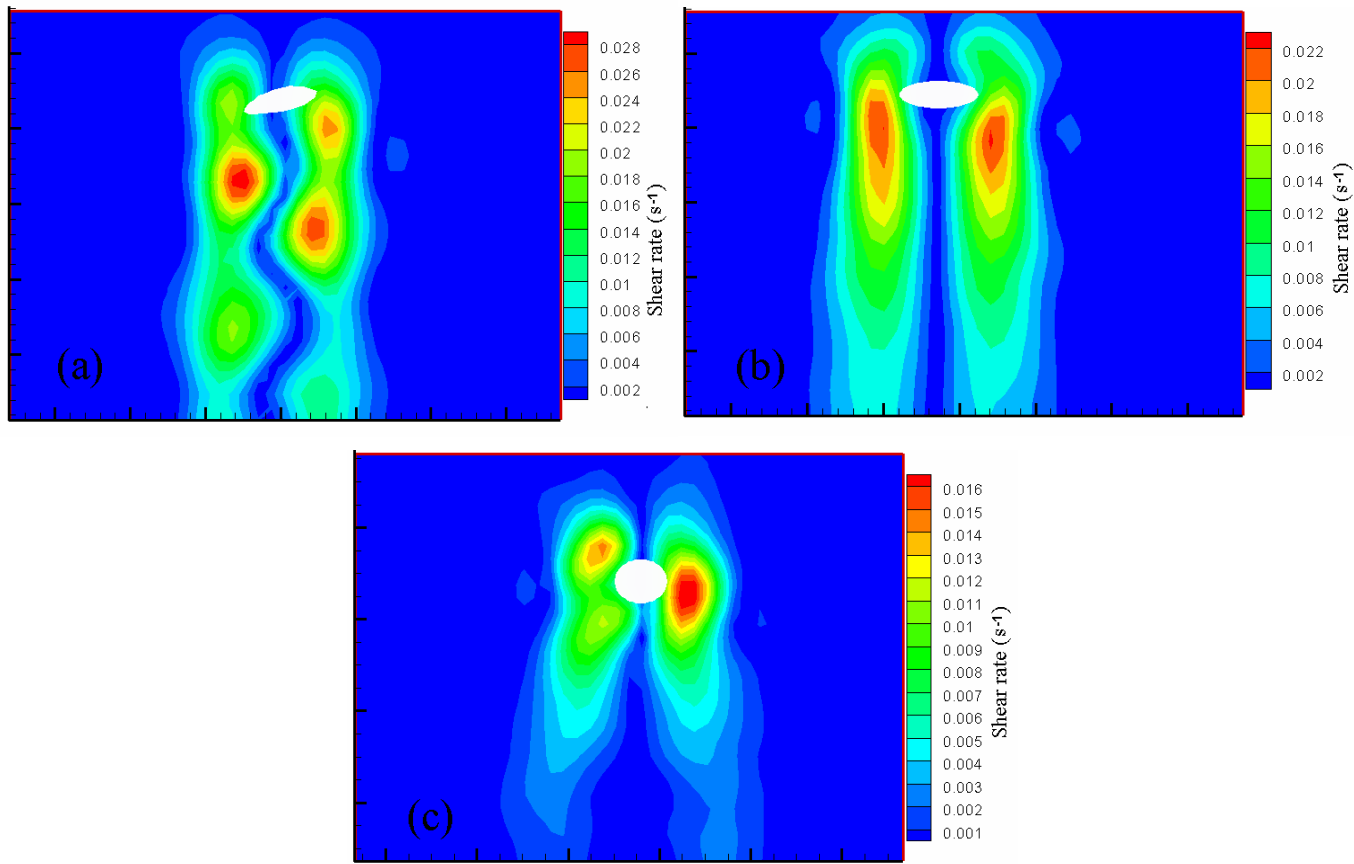

Figure 9: Shear rate distribution in CMC solutions. (a) $0.2 \% \mathrm{CMC}, \mathrm{d}_{\mathrm{e}}=8.0 \mathrm{~mm}$; (b) $0.6 \% \mathrm{CMC}, \mathrm{d}_{\mathrm{e}}=8.2 \mathrm{~mm}$; (c) $1.0 \% \mathrm{CMC}, \mathrm{d}_{\mathrm{e}}=8.1 \mathrm{~mm}$.

\section{CONCLUSIONS}

The flow field and viscosity distribution of the liquid around a single bubble rising in a nonNewtonian fluid was studied by the PIV technique. Within the range of experimental conditions, the flow field of the liquid around a single rising bubble at different concentrations of CMC reveals a similar structure: the rising bubble pushed the liquid in front of it (up-flow) and then the liquid flowed back into the bubble wake, forming a pseudo-plug flow. A spiral vortex rope appeared around the wake of the rising bubble at low concentrations of CMC. The spiral vortex rope disappeared gradually with the appearance of a vortex ring when the concentration of CMC solutions increased. The viscosity field was closely related to the flow field due to the rheological characteristics of non-Newtonian fluids. The large relative velocity between the liquid of the wake and the bulk liquid led to a hollow cylindrial low-viscosity region. Conversely, the small relative velocity of the internal liquids of the wake led to a high viscosity region. The concentration of the CMC solution had a remarkable influence on the viscosity distribution. The minimal specific viscosity decreased with the increase of the concentration of $\mathrm{CMC}$ due to the strong shear-thinning effect. The shear stress property is principally responsible for the viscosity change of a non-Newtonian fluid, so the distribution of shear stress could be directly reflected to the viscosity distribution. The study of the viscosity distribution around a bubble rising in nonNewtonian fluids should be helpful to further understand the behavior of bubble motion and the interaction between bubbles.

\section{ACKNOWLEDGEMENTS}

The authors gratefully acknowledge the financial support of the National Natural Science Foundation of China (No. 21076139, 20476073), the State Key Laboratory of Chemical Engineering (SKL-ChE08B03), and the Programs of Introducing Talents of Discipline to Universities (B06006).

\section{NOMENCLATURE}

$\mathrm{d}_{\mathrm{e}} \quad$ volume equivalent bubble

$\mathrm{m}$ diameter

D unit of molecular weight

$\mathrm{K}$ consistency index

$\mathrm{n} \quad$ flow index Reynolds number of the dimensionless 


\begin{tabular}{|c|c|c|}
\hline$S$ & Carreau model parameter & \\
\hline $\mathrm{U}$ & bubble velocity & \\
\hline $\mathrm{U}_{\mathrm{x}}$ & $\begin{array}{l}\text { liquid velocity component in } \\
\text { the } \mathrm{x} \text { direction }\end{array}$ & \\
\hline $\mathrm{U}_{\mathrm{y}}$ & $\begin{array}{l}\text { liquid velocity component in } \\
\text { the y direction }\end{array}$ & \\
\hline $\mathrm{X}$ & radial coordinate & \\
\hline$\Delta \mathrm{x}$ & $\begin{array}{l}\text { the length in the } x \text { direction } \\
\text { at the calculated grid point } \\
(i, j)\end{array}$ & \\
\hline y & axial coordinate & \\
\hline$\Delta y$ & $\begin{array}{l}\text { the length in the y direction } \\
\text { at the calculated grid point } \\
(i, j)\end{array}$ & \\
\hline
\end{tabular}

\section{Greek Symbols}

$\begin{array}{llr}\dot{\gamma} & \text { shear rate } & \mathrm{s}^{-1} \\ \eta & \text { specific viscosity } & \text { dimensionless } \\ \eta_{0} & \text { zero-shear viscosity } & \mathrm{Pa} \cdot \mathrm{s} \\ \eta(\dot{\gamma}) & \text { apparent viscosity } & \mathrm{Pa} \cdot \mathrm{s} \\ \lambda & \text { Carreau model parameter } & \mathrm{s} \\ \tau & \text { shear stress } & \mathrm{Pa} \\ \rho & \text { density } & \mathrm{kg} \cdot \mathrm{m}^{-3} \\ \sigma & \text { surface force } & \mathrm{N} \cdot \mathrm{m}^{-1}\end{array}$

\section{REFERENCES}

Allen, D. G., Robinson, C. W., Comments on the communication: on the calculation of shear rate and apparent viscosity in air lift and bubble column reactors. Biotechnology and Bioengineering, 38, (2), 212-216 (1991).

Al-Masry, W. A., Effect of scale-up on average shear rates for aerated non-Newtonian liquids in external loop airlift reactors. Biotechnology and Bioengineering, 62, (4), 494-498 (1999).

Brücker, C., Structure and dynamics of the wake of bubbles and its relevance for bubble interaction. Physics of Fluids, 11, (7), 1781-1796 (1999).

Carreau, P. J., Rheological equations from molecular network theories. Journal of Rheology, 16, (1), 99-127 (1972).

Chen, R. C., Chou, I. S., Wake structure of a single bubble rising in a two-dimensional column. Experimental Thermal and Fluid Science, 17, (3), 165-178 (1998).

Clift, R., Grace, J. R., Weber, M. E., Bubbles, Drops, and Particle. Academic Press, New York (1978).

Davies, R. M., Taylor, G. I., The mechanics of large bubbles rising through extended liquids and through liquids in tube. Proceedings of the Royal Society of London, 200, (A), 375-390 (1950).

Funfschilling, D., Li, H. Z., Flow of non-Newtonian fluids around bubbles: PIV measurements and birefringence visualization. Chemical Engineering Science, 56, (3), 1137-1141 (2001).

Hassan, Y. A., Oritz-Villafuerte, J., Schmidl, W. D., Three dimensional measurements of single bubble dynamics in a small diameter pipe using stereoscopic particle image velocimetry. International Journal of Multiphase Flow, 27, (5), 817-842 (2001).

Kawase, Y., Kumagai, T., Apparent viscosity for non-Newtonian fermentation media in bioreactors. Bioprocess and Biosystems Engineering, 7, (1-2), 25-28 (1991).

Kulkarni, A. A., Joshi, J. B., Bubble formation and bubble rise velocity in gas-liquid system: a review. Industrial \& Engineering Chemistry Research, 44, (16), 5873-5931 (2005).

Lance, M., Bataille, J., Turbulence in the liquid phase of a uniform bubbly air-water flow. Journal of Fluid Mechanics, 222, 95-118 (1991).

Li, H. Z., Mouline, Y., Funfschilling, D., Marchal, P., Evidence for in-line bubble interactions in non-Newtonian fluids. Chemical Engineering Science, 53, (12), 2219-2230 (1998).

Mandal, A., Characterization of gas-liquid parameters in a down-flow jet loop bubble column. Brazilian Journal of Chemical Engineering, 27, (2), 253264 (2010).

Moore, D., The rise of a gas bubble in a viscous liquid. Journal of Fluid Mechanics, 6, (1), 113130 (1958).

Ribeiro Jr., C. P., Lage, P. L. C., Experimental study on bubble size distributions in a direct-contact evaporator. Brazilian Journal of Chemical Engineering, 21, (1), 69-81 (2004).

Rodrigue, D., A general correlation for the rise velocity of single gas bubbles. The Canadian Journal of Chemical Engineering, 82, 382-386 (2004).

Schowalter, W. R., The application of boundary-layer theory to power law pseudoplastic fluids: similarity solutions. AIChE Journal, 6, (1), 24-28 (1960).

Toshiyuki, S., Masao, W., Tohru, F., Effects of viscosity on coalescence of a bubble upon impact with a free surface. Chemical Engineering Science, 60, (19), 5372-5384 (2005).

Wang, H., Zhang, Z. Y., Yang, Y. M., Zhang, H. S., Viscosity effects on the behavior of a rising bubble. Journal of Hydrodynamics, 22, (1), 81-89 (2010). 
Zenit, R., Magnaudet, J., Measurements of the streamwise vorticity in the wake of an oscillating bubble. International Journal of Multiphase Flow, 35, (2), 195-203 (2009).

Zhang, L., Yang, C., Mao, Z. S., Numerical simulation of a bubble rising in shear-thinning fluids. Journal of Non-Newtonian Fluid Mechanics, 165, (11-12), 555-567 (2010).

Zhao, J., Zhang X. Q., Yu, J. T., $\mathrm{O}_{2}$ transfer to pseudoplastic fermentation broths in air-lift reactors with different inner designs. Biotechnology Techniques, 12, (10), 729-732 (1998). 